\title{
Turbulence and flows in the plasma boundary of snowflake magnetic configurations
}

\author{
M. Giacomin, L. N. Stenger and P. Ricci \\ Ecole Polytechnique Fédérale de Lausanne (EPFL), Swiss Plasma Center (SPC), CH-1015 Lausanne, \\ Switzerland \\ E-mail: maurizio.giacomin@epfl.ch
}

\begin{abstract}
Results of 3D, flux-driven, global, two-fluid turbulence simulations are presented for the first time in snowflake configurations, including the ideal snowflake, snowflake plus and snowflake minus configurations. The analysis is focused on the distribution of the heat flux along the four divertor legs in the sheath-limited regime and on the mechanisms that activate the four strike points. A steady $\mathbf{E} \times \mathbf{B}$ equilibrium flow in the X-point region is found to play an important role for the activation of the four strike points, especially in the ideal snowflake case. Turbulent transport, observed in the region between the two separatrices for the snowflake minus configuration when the secondary $\mathrm{X}$-point is on the low-field side, contributes to the redistribution of heat flux among the strike points.
\end{abstract}

Keywords: heat flux, plasma turbulence, scrape-off layer, snowflake, GBS

Submitted to: Nucl. Fusion

Power exhaust through the scrape-off layer (SOL) in fusion reactors is expected to be significantly higher than in ITER $[1,2]$. This questions the extrapolation of the ITER exhaust solution to these devices. Among the alternative exhaust configurations considered to mitigate the heat vessel loads in fusion reactors [3], we focus here on the snowflake (SF) [4]. The SF features a second-order null of the poloidal magnetic field, i.e. a point where all the first and second derivatives of the poloidal flux function vanish. As a consequence, the null-point is connected to the vessel wall through four legs, which define four strike points. It has been observed that these four strike points can distribute the exhaust power on a larger area than standard divertor configurations that feature two strike points [4].

SF configurations are obtained experimentally by generating two first-order X-points close to each other. When the two $\mathrm{X}$-points coincide, a second-order null point is obtained. However, in practice, the two Xpoints never coincide perfectly: The $\mathrm{X}$-point associated with the separatrix that encloses the plasma is denoted as primary, while the other, the secondary $\mathrm{X}$-point, lays either in the private flux region of the primary X-point or in the common flux region. The first configuration is denoted as the snowflake plus ( $\mathrm{SF}+)$, the latter as snowflake minus ( $\mathrm{SF}-$ ), while the configuration with the two X-points coinciding is usually referred to as the ideal SF. In the SF-, the secondary X-point can be positioned either on the high-field side (HFS SF-) or on the low-field side (LFS SF-) with respect to the primary separatrix (see Fig. 1 for examples of these configurations). All these configurations have been experimentally investigated in the TCV [5-8], NSTX $[9,10]$, and DIII-D [11] tokamaks.

Experimental measurements in the SF have shown a reduction of the peak heat fluxes compared to similar single-null (SN) configurations [6]. This was explained as the result of the presence of an additional cross-field transport channel into the private flux region. In Ref. [8], the effective SOL width in the LFS SF-, inferred from the measured power repartition between the two SOL regions created by the secondary $\mathrm{X}$-point, was twice as large as that measured by a reciprocating probe at the outboard midplane, an observation interpreted as the result of an enhanced cross-field turbulent transport in the nullregion. Numerical simulations of SF configurations, carried out by means of the EMC3-Eirene $[12,13]$ and the SOLPS [14] codes, are unable to reproduce these experimental observations and predict a negligible heat flux on the strike points connected to the secondary X-point [12]. These simulations assume constant diffusion parameters and neglect equilibrium drifts. The discrepancies between experimental observations and simulations call for detailed investigation of the presence of mechanisms leading to an enhanced crossfield transport. By evolving self-consistently the turbulent and the equilibrium cross-field transport, 


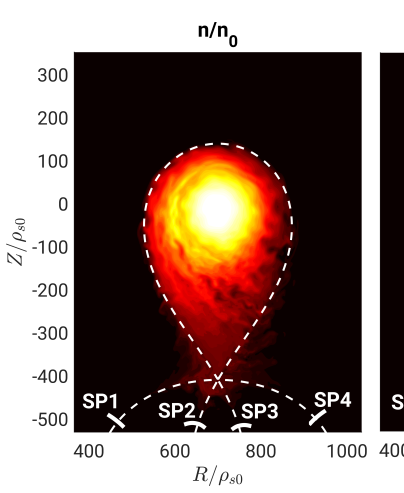

(a) Ideal $S F$

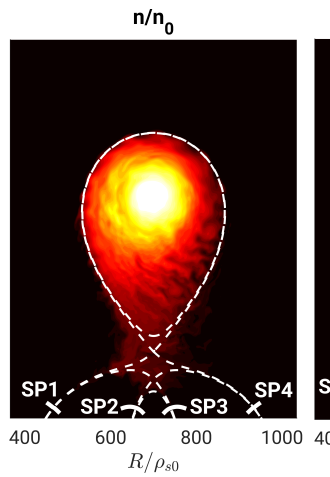

(b) $S F+$

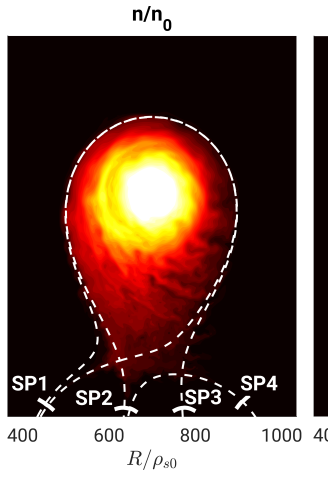

(c) $L F S S F-$

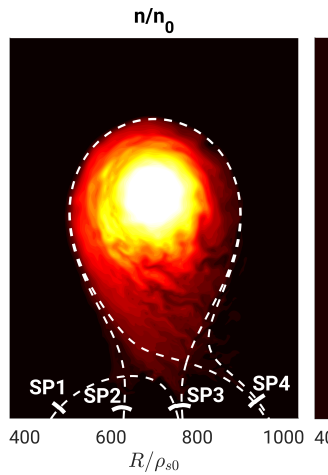

(d) $H F S S F-$

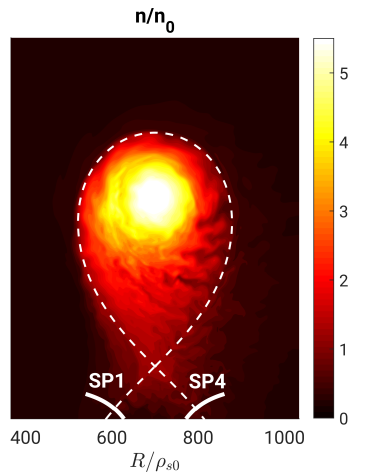

(e) $S N$

Figure 1: Typical snapshot of density, n, on a poloidal plane for the ideal SF (a), the SF+ (b), the LFS SF(c), the HFS SF- (d), and the $S N(e)$. The dashed white line indicates the separatrices and the solid white lines the locations where the parallel heat flux is evaluated. Starting from the HFS, we label the strike points as SP1, SP2, SP3 and SP4 for the SF configurations, and SP1 and SP4 for the SN configuration.

first-principles global turbulence simulations can point out the existence of regions where the perpendicular transport is strong and the mechanisms behind it.

In the present Letter, for the first time, firstprinciples turbulent simulations in the SF configurations are presented. The ideal $\mathrm{SF}$, the $\mathrm{SF}+$, the LFS SF-, and the HFS SF- are analysed, revealing a complex interplay between turbulence and steady state flows. The simulations are carried out by using GBS [15-17], a three-dimensional, flux-driven, firstprinciples simulation code that has been developed to study the turbulence in the plasma boundary of tokamak devices. Similarly to other turbulent codes (BOUT ++ [18], GDB [19], GRILLIX [20], HESEL [21], and TOKAM3X [22]), GBS evolves the driftreduced Braginskii's equations (see e.g. Ref. [23]), a set of two-fluid equations valid to describe phenomena occurring on time scales longer than $1 / \Omega_{c i}$, with $\Omega_{c i}=e B /\left(\mathrm{cm}_{i}\right)$ the ion cyclotron frequency, perpendicular length scales longer than the ion Larmor radius, and parallel length scales longer than the mean free path. Under these assumptions, the ion fluid velocity $\mathbf{v}_{\mathbf{i}}$ is written as a sum of the $\mathbf{E} \times \mathbf{B}$ drift velocity, $\mathbf{v}_{\mathbf{E}}=-c \nabla \phi \times \mathbf{B} / B^{2}$, the ion diamagnetic drift velocity, $\mathbf{v}_{* \mathbf{i}}=c \mathbf{B} \times \nabla p_{i} /\left(e n B^{2}\right)$, the polarization drift velocity, $\mathbf{v}_{\mathbf{p o l}}$, and the parallel velocity, $v_{\| i}$, i.e. $\mathbf{v}_{\mathbf{i}}=\mathbf{v}_{\mathbf{E}}+\mathbf{v}_{* \mathbf{i}}+\mathbf{v}_{\mathbf{p o l}}+v_{\| i} \mathbf{B} / B$. The same applies to the electron fluid velocity, $\mathbf{v}_{\mathbf{e}}=\mathbf{v}_{\mathbf{E}}+\mathbf{v}_{* \mathbf{e}}+v_{\| e} \mathbf{B} / B$, where the electron polarization drift is neglected [15]. The description of the model implemented in GBS is summarized in Ref. [16]. For simplicity, here we neglect the neutral dynamics, therefore considering the sheath-limited regime. We also neglect the electromagnetic effects and we apply the Boussinesq approximation $[15,24-26]$ in the evaluation of the divergence of the polarization current. The effect of the Boussi-

nesq approximation has been discussed in Refs. [24,27], showing that it has a negligible effect on SOL turbulence. Finally, we consider the large aspect-ratio limit $[17,28]$. As a result, the model equations evolved by GBS are the following [15]:

$$
\begin{aligned}
\frac{\partial n}{\partial t} & =-\frac{c}{B}[\phi, n]+\frac{2 c}{e B}\left[C\left(p_{e}\right)-e n C(\phi)\right] \\
& -\nabla_{\|}\left(n v_{\| e}\right)+D_{n} \nabla_{\perp}^{2} n+S_{n} \\
\frac{\partial \omega}{\partial t} & =-\frac{c}{B}[\phi, \omega]-v_{\| i} \nabla_{\|} \omega+\frac{B \Omega_{c i}}{c e n} \nabla_{\|} j_{\|} \\
& +\frac{2 \Omega_{c i}}{e n} C\left(p_{e}+\tau p_{i}\right)+\frac{\Omega_{c i}}{3 e n} C\left(G_{i}\right)+D_{\omega} \nabla_{\perp}^{2} \omega \\
\frac{\partial v_{\| e}}{\partial t} & =-\frac{c}{B}\left[\phi, v_{\| e}\right]-v_{\| e} \nabla_{\|} v_{\| e} \\
& +\frac{e}{m_{e}}\left(\frac{j_{\|}}{\sigma_{\|}}+\nabla_{\|} \phi-\frac{1}{e n} \nabla_{\|} p_{e}-\frac{0.71}{e} \nabla_{\|} T_{e}\right) \\
& -\frac{2}{3 n m_{e}} \nabla_{\|} G_{e}+D_{v_{\| e}} \nabla_{\perp}^{2} v_{\| e}, \\
\frac{\partial v_{\| i}}{\partial t} & =-\frac{c}{B}\left[\phi, v_{\| i}\right]-v_{\| i} \nabla_{\|} v_{\| i}-\frac{1}{m_{i} n} \nabla_{\|}\left(p_{e}+\tau p_{i}\right) \\
& -\frac{2}{3 n m_{i}} \nabla_{\|} G_{i}+D_{v_{\| i}} \nabla_{\perp}^{2} v_{\| i} \\
\frac{\partial T_{e}}{\partial t} & =-\frac{c}{B}\left[\phi, T_{e}\right]-v_{\| e} \nabla_{\|} T_{e} \\
& +\frac{2}{3} T_{e}\left[0.71 \nabla_{\|} v_{\| i}-1.71 \nabla_{\|} v_{\| e}\right] \\
& +\frac{2}{3} T_{e}\left[0.71\left(v_{\| i}-v_{\| e}\right) \frac{\nabla_{\|} n}{n}\right] \\
& +\frac{4}{3} \frac{c T_{e}}{e B}\left[\frac{7}{2} C\left(T_{e}\right)+\frac{T_{e}}{n} C(n)-C(\phi)\right] \\
& +\chi_{\perp e} \nabla_{\perp}^{2} T_{e}+\chi_{\| e} \nabla_{\|}^{2} T_{e}+S_{T_{e}} \\
\frac{\partial T_{i}}{\partial t} & =-\frac{c}{B}\left[\phi, T_{i}\right]-v_{\| i} \nabla_{\|} T_{i} \\
& =-1(5)
\end{aligned}
$$




$$
\begin{aligned}
& +\frac{4}{3} \frac{c T_{i}}{e B}\left[C\left(T_{e}\right)+\frac{T_{e}}{n} C(n)-C(\phi)\right]-\frac{10}{3} \frac{c T_{i}}{e B} C\left(T_{i}\right) \\
& +\frac{2}{3} T_{i}\left(v_{\| i}-v_{\| e}\right) \frac{\nabla_{\|} n}{n}-\frac{2}{3} T_{i} \nabla_{\|} v_{\| e} \\
& +\chi_{\perp i} \nabla_{\perp}^{2} T_{i}+\chi_{\| i} \nabla_{\|}^{2} T_{i}+S_{T_{i}} \\
\nabla_{\perp}^{2} \phi & =\omega-\frac{\nabla_{\perp}^{2} T_{i}}{e} .
\end{aligned}
$$

By normalizing Eqs. (1-7), three dimensionless parameters that regulate the system dynamics are identified: the normalized ion sound Larmor radius, $\rho_{*}=$ $\rho_{s 0} / R_{0}$, the ion to electron temperature ratio, $\tau=$ $T_{i 0} / T_{e 0}$, and the normalized Spitzer resistivity, $\nu=$ $e^{2} n_{0} R_{0} /\left(m_{i} c_{s 0} \sigma_{\|}\right)=\nu_{0} T_{e}^{-3 / 2}$, with

$\sigma_{\|}=1.96 \frac{n e^{2} \tau_{e}}{m_{e}}=\left(\frac{5.88}{4 \sqrt{2 \pi}} \frac{T_{e 0}^{3 / 2}}{\lambda \sqrt{m_{e} e}}\right) \frac{T_{e}^{3 / 2}}{T_{e 0}^{3 / 2}}$,

$\nu_{0}=\frac{4 \sqrt{2 \pi}}{5.88} \frac{\sqrt{m_{e}} R_{0} n_{0} \lambda e^{5 / 2}}{m_{i} c_{s 0} T_{e 0}^{3 / 2}}$,

$\rho_{s 0}=c_{s 0} / \Omega_{c i}$, and $c_{s 0}=\sqrt{T_{e 0} / m_{i}}$, where $n_{0}, T_{e 0}$, and $T_{i 0}$ are the reference density, electron temperature, ion temperature, respectively, and $\lambda$ is the Coulomb logarithm. The source terms in the density and temperature equations, $S_{n}$ and $S_{T}$, are added to fuel and heat the plasma.

The toroidally symmetric equilibrium magnetic field is written in terms of the poloidal magnetic flux $\psi$ as

$$
\mathbf{B}=F(\psi) \nabla \varphi+\nabla \psi \times \nabla \varphi,
$$

where $\varphi$ is the toroidal angle. The first term on the right-hand side of Eq. (10) is the toroidal component of the equilibrium magnetic field, with $F(\psi)$ a scalar function of $\psi$, proportional to the enclosed poloidal current, while the second term is the poloidal component of the equilibrium magnetic field. The poloidal flux is a function of the distance from the tokamak axis of symmetry $R$ and of the vertical coordinate $Z, \psi=\psi(R, Z)$. In Eqs. (1-7) we introduce the $\mathbf{E} \times \mathbf{B}$ convective term $[g, f]=\mathbf{b} \cdot(\nabla g \times \nabla f)$, the curvature operator $C(f)=B / 2[\nabla \times(\mathbf{b} / B)] \cdot \nabla f$, the parallel gradient $\nabla_{\|} f=\mathbf{b} \cdot \nabla f$, and the perpendicular Laplacian $\nabla_{\perp}^{2} f=\nabla^{2} f-\nabla \cdot[\mathbf{b}(\mathbf{b} \cdot \nabla f)]$, being $\mathbf{b}=$ $\mathbf{B} / B$ the unit vector of the magnetic field. The numerical implementation of Eqs. (1-7), including the definition of the gyroviscous terms $G_{e, i}$ and other dissipative contributions, is detailed in Ref. [17]. We note that GBS solves Eqs. (1-7) on a nonfield aligned grid, which allows for simulations in arbitrary magnetic configurations, by using a fourthorder finite differences scheme. A convergence study performed in Ref. [17] shows that the order of convergence is correctly retrieved with the refining of the grid resolution. (As an aside, we would like to mention that another approach to the simulation of
SF configurations is being considered by GRILLIX, based on a flux-coordinate independent approach [29]). The poloidal cross section has a rectangular shape with size $L_{R}$ and $L_{Z}$, in the radial and vertical direction, and encompasses the whole tokamak, with the tokamak core fluctuations having approximately a $1 \%$ amplitude, in agreement with experimental observations [30]. This avoids the need of imposing a set of arbitrary boundary conditions with the core, as opposed to typical simulations of the plasma dynamics in the tokamak boundary. The density and the temperature sources are analytical functions of $\psi(R, Z)$ and they are toroidally uniform. More precisely, the particle source,

$$
S_{n}=S_{n 0} \exp \left(-\frac{\left(\psi(R, Z)-\psi_{n}\right)^{2}}{\Delta_{n}^{2}}\right),
$$

is centered around the flux surface $\psi_{n}$, located inside the last closed flux surface. The temperature source,

$$
S_{T}=\frac{S_{T 0}}{2}\left[\tanh \left(-\frac{\psi(R, Z)-\psi_{t}}{\Delta_{T}}\right)+1\right],
$$

approaches $S_{T 0}$ in the region inside the flux surface $\psi_{t}$, located in the core, and vanishes moving towards the periphery. Magnetic pre-sheath boundary conditions, derived in Ref. [31], are applied at the divertor plates.

The magnetic poloidal flux $\psi$ is analytically obtained by considering three straight current filaments and a current density with Gaussian profile, which mimics the plasma current. The position of the filaments and the plasma current is shown in Fig. 2. The plasma current is centered at $\left(R_{p}, Z_{p}\right)$, its integral value is $I_{p}$ and its width $\sigma$. We denote $\left(R_{i}, Z_{i}\right)$ and $I_{i}$, with $i=1,2,3$, the position of the three filaments and their current, respectively. The position of the filaments are chosen, for instance, to develop a left-right symmetric equilibrium in the ideal SF case. This imposes $R_{1}+R_{2}=2 R_{p}, R_{3}=R_{p}, I_{1}=I_{2} \equiv \alpha I_{p}$ and $Z_{1}=Z_{2}$. The distance between the first and the second filament, $d \equiv R_{2}-R_{1}$, and $\alpha$ are derived by following the procedure described in Ref. [4]. In practice, we impose $\nabla \psi, \partial_{R} \nabla \psi$ and $\partial_{Z} \nabla \psi$ to vanish at the second-order X-point and we obtain

$$
\begin{aligned}
& \alpha=-\frac{Z_{1}^{\prime} \sigma^{2}\left[Z_{3}^{\prime}-\left(\beta Z_{p}^{\prime}+Z_{3}^{\prime}\right) \exp \left(Z_{p}^{\prime 2} / 2 \sigma^{2}\right)\right]^{2}}{\exp \left(Z_{p}^{\prime 2} / \sigma^{2}\right)\left[K_{1}-K_{2} \exp \left(-Z_{p}^{\prime 2} / 2 \sigma^{2}\right)\right]} \\
& d=-\frac{2 Z_{1}^{\prime}\left[K_{3} \exp \left(Z_{p}^{\prime 2} / 2 \sigma^{2}\right)-K_{4}\right]^{1 / 2}}{\left[K_{1} \exp \left(Z_{p}^{\prime 2} / 2 \sigma^{2}\right)-K_{2}\right]^{1 / 2}}
\end{aligned}
$$

where $\beta=I_{3} / I_{p}$,

$$
\begin{aligned}
& K_{1}=\sigma^{2}\left[Z_{3}^{\prime 2}\left(Z_{p}^{\prime}+Z_{1}^{\prime}\right)+\beta Z_{p}^{\prime 2}\left(Z_{1}^{\prime}+Z_{3}^{\prime}\right)\right], \\
& K_{2}=Z_{3}^{\prime 2}\left(Z_{p}^{\prime 2} Z_{1}^{\prime}+Z_{p}^{\prime} \sigma^{2}+Z_{1}^{\prime} \sigma^{2}\right), \\
& K_{3}=\sigma^{2}\left[\beta Z_{p}^{\prime 2}\left(Z_{3}^{\prime}-Z_{1}^{\prime}\right)+Z_{3}^{\prime 2}\left(Z_{p}^{\prime}-Z_{1}^{\prime}\right)\right], \\
& K_{4}=Z_{3}^{\prime 2}\left[\sigma^{2}\left(Z_{p}^{\prime}-Z_{1}^{\prime}\right)-Z_{p}^{\prime 2} Z_{1}^{\prime}\right],
\end{aligned}
$$




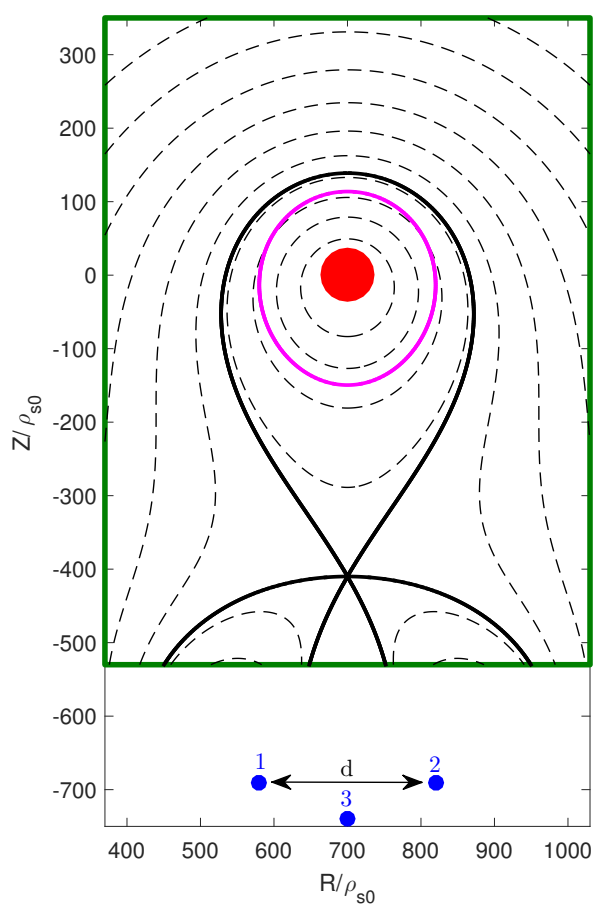

Figure 2: Contour plot of the poloidal flux function, $\psi$, in the ideal SF configuration implemented in GBS (black dashed line). The separatrix is shown as a solid black line. The GBS boundary domain is indicated by a solid green line. The three blue circles represent the position of the current filaments, located outside the simulation domain, responsible of creating the SF configuration, while the red circle represents the center of the plasma current. The flux surface $\psi=\psi_{n}=\psi_{t}$ is shown as a solid magenta line.

with $Z_{p}^{\prime}=Z_{p}-Z_{X}, Z_{1}^{\prime}=Z_{1}-Z_{X}$, and $Z_{3}^{\prime}=Z_{3}-Z_{X}$, being $Z_{X}$ the vertical position of the X-point. The $\mathrm{SF}+$ and $\mathrm{SF}-$ configurations are obtained by varying the current of the $i=1$ and $i=2$ filaments.

The simulations we present are carried out with the following parameters: $\rho_{*}^{-1}=700, a / R_{0}=0.25$, $\nu_{0}=0.1, \tau=1, L_{R}=660 \rho_{s 0}, L_{Z}=880 \rho_{s 0}$, $S_{n 0} / n_{0}=0.3 c_{s 0} / R_{0}, \Delta_{n}=900, S_{T 0} / T_{e 0}=0.3$ $c_{s 0} / R_{0}, \Delta_{T}=800, \sigma=63.3 \rho_{s 0}$, and $I_{p} /\left(e n_{0} c_{s 0}\right)=$ 11.4. The position of the flux surface $\psi=\psi_{n}=\psi_{t}$ is shown in Fig. 2. We choose $Z_{p}^{\prime}=410 \rho_{s 0}, Z_{1}^{\prime}=$ $-280 \rho_{s 0}, Z_{3}^{\prime}=-330 \rho_{s 0}$, and $\beta=-7.0$ for all the SF configurations. With this choice of parameters, we have the safety factors $q_{0} \simeq 1$ at the magnetic axis and $q_{95} \simeq 5$. From Eqs. (13-14) one evaluates $\alpha=3.91$ and $d=240 \rho_{s 0}$ for the ideal SF. The values of the current in the three filaments for the four configurations are reported in Table 1 . The ion- $\nabla B$ drift direction points upwards (unfavourable for H-mode access). Regarding the numerical parameters, in all cases, the grid used is $N_{R} \times N_{Z} \times N_{\varphi}=240 \times 320 \times 80$ and the time-step is

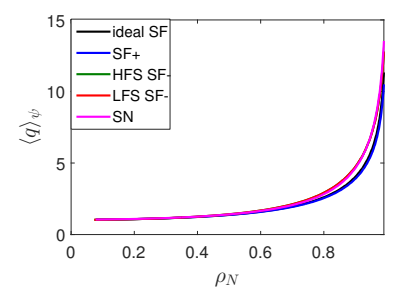

(a) Safety factor

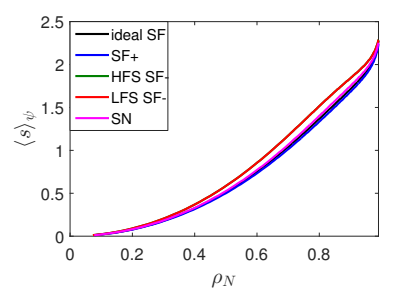

(b) Magnetic shear

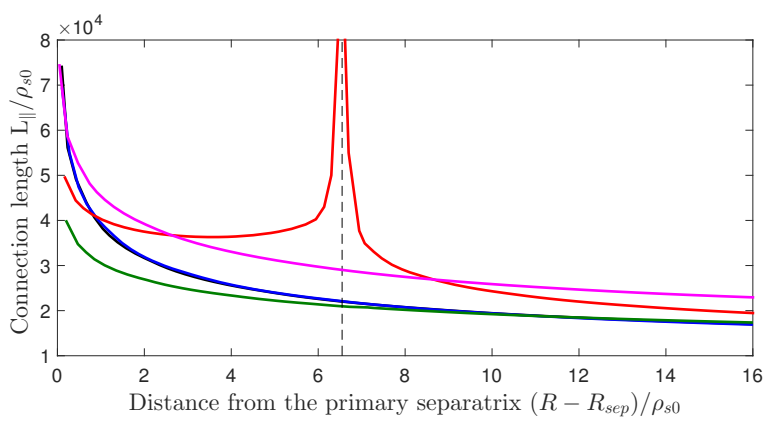

(c) Low-field side connection length

Figure 3: Flux-surface averaged safety factor, $\langle q\rangle_{\psi}$, (a) and magnetic shear, $\langle s\rangle_{\psi}$, (b) inside the separatrix for all the considered configurations as a function of $\rho_{N}=\sqrt{\left(\psi-\psi_{0}\right) /\left(\psi_{L C S F}-\psi_{0}\right)}$, where $\psi_{L C F S}$ and $\psi_{0}$ are the poloidal flux values at the last closed flux surface and at the magnetic axis, respectively. The low-field side connection length is shown in panel (c). The vertical dashed line at the distance $R-R_{\text {sep }} \simeq$ $7 \rho_{s 0}$ from the primary separatrix, where $L_{\|}$diverges, corresponds to the location of the secondary separatrix of the LFS SF-.

$2 \times 10^{-5} R_{0} / c_{s 0}$. The analysis that follows is performed when a quasi-steady state is reached, where sources, turbulent transport and losses at the vessel balance each other. A snapshot of the density on a poloidal plane is shown in Fig. 1 for all the SF configurations.

In order to evaluate the effect of the SF on the power exhaust, we consider a SN configuration with core conditions similar to the ideal SF. The SN configuration is obtained by using the same set of filaments, and the same physical and numerical parameters, as in the SF simulations, but $\alpha=0.3$, $d=1000 \rho_{s 0}, Z_{p}^{\prime}=410 \rho_{s 0}, Z_{1}^{\prime}=0, Z_{3}^{\prime}=-480 \rho_{s 0}$, and $\beta=1.17$. The value of the current in the three filaments for the $\mathrm{SN}$ is also reported in Table 1. In Fig. 1 (e) a typical snapshot of the density in the SN configuration is shown.

The SF and SN configurations are designed to have similar flux-averaged safety factor and magnetic shear profiles in the core [see Fig. 3 (a) and (b)]. The SOL width at the LFS midplane computed from the pressure profile is similar for all the configurations and its value is approximately $10 \rho_{s 0}$. 
Table 1: Values of the current in the three filaments normalized to the plasma current for all the considered configurations. Negative currents flow in the direction opposite to the plasma current.

\begin{tabular}{llll}
\hline Configuration & $\mathbf{I}_{\mathbf{1}} / \mathbf{I}_{\mathbf{p}}$ & $\mathbf{I}_{\mathbf{2}} / \mathbf{I}_{\mathbf{p}}$ & $\mathbf{I}_{\mathbf{3}} / \mathbf{I}_{\mathbf{p}}$ \\
\hline Ideal SF & 3.91 & 3.91 & -7.00 \\
SF+ & 3.94 & 3.94 & -7.00 \\
HFS SF- & 3.91 & 3.72 & -7.00 \\
LFS SF- & 3.72 & 3.91 & -7.00 \\
SN & 0.3 & 0.3 & 1.17 \\
\hline
\end{tabular}

Table 2: Fraction of the deposited power on the different strike points for all the considered magnetic configurations. See Fig. 1 for the position of the strike points.

\begin{tabular}{lrrrr}
\hline & SP1 & SP2 & SP3 & SP4 \\
\hline Ideal SF & $28 \%$ & $20 \%$ & $18 \%$ & $34 \%$ \\
SF+ & $30 \%$ & $14 \%$ & $18 \%$ & $38 \%$ \\
LFS SF- & $28 \%$ & $53 \%$ & $9 \%$ & $10 \%$ \\
HFS SF- & $6 \%$ & $8 \%$ & $60 \%$ & $26 \%$ \\
SN & $46 \%$ & - & - & $54 \%$ \\
\hline
\end{tabular}

This recalls experimental observations that show a weak dependence of the upstream SOL width on the different magnetic configurations $[6,8]$. The low-field side (LFS) connection length, $L_{\|}$, defined as the length of a magnetic field line connecting the outer midplane to the LFS target, is displayed in Fig. 3 (c) for all the configurations. The SN configuration has a longer LFS connection length than the ideal $\mathrm{SF}$, the $\mathrm{SF}+$, and the HFS SF - within a distance from the separatrix larger than approximately $0.1 \rho_{s 0}$, which is considerably shorter than the SOL width. This is typical for presentday devices, but the distance from the separatrix at which the $L_{\|}$of a SF configuration is equal to $L_{\|}$of a comparable SN configuration in fusion reactors is expected to be larger than the SOL width [6]. The connection length in the LFS SF- diverges at the location of the secondary separatrix, whose distance from the primary separatrix at the LFS midplane is approximately $7 \rho_{s 0}$.

In the present Letter, we focus on the distribution of the equilibrium parallel heat flux among the four legs of the ideal SF, the SF+, the LFS SF-, and the HFS $\mathrm{SF}-$. Since we consider the sheath-limited regime, the parallel heat flux is dominated by the parallel transport, $q_{\|}=\overline{p_{e} v_{\| e}}$, (we write any quantity $f$ as a sum of the time- and toroidal-averaged component $\bar{f}$, denoted here as equilibrium, and the fluctuating component $\tilde{f}$, i.e. $f=\bar{f}+\tilde{f})$. The $q_{\|}$flux is proportional to the power reaching the wall through the sheath heat transmission coefficient [32]. In Fig. 4, we show $q_{\|}$in the proximity of the four strike points, which are denoted, from the HFS to the LFS, as SP1, SP2, SP3, and SP4, and whose position is displayed in Fig. 1. The distance from the separatrix is evaluated at the midplane in order to remove the effect of the flux expansion at the target, which is different for all the configurations and could be compensated by a poloidal tilt of the target in a dedicated device. The fraction of the deposited power on the strike points is listed in Table 2. We now analyse the mechanisms behind the distribution of the heat flux over the four legs in all configurations.

In the ideal $\mathrm{SF}$, in absence of perpendicular transport, we expect that the heat flux flows along the magnetic field lines to SP1 and SP4. On the other hand, we notice that the integrated heat flux redistributes quite homogeneously (see Table 2) among the four strike points. The activation of the inner legs (SP2 and SP3) is due to a convection cell induced by the equilibrium $\mathbf{E} \times \mathbf{B}$ drift, $\mathbf{q}_{\mathrm{E}}^{\mathrm{eq}}=\bar{p}_{\mathrm{e}} \overline{\mathbf{v}}_{\mathrm{E}}$, present in the region around the null-point (the turbulent flux is negligible in the null-point region as observed experimentally in the SN configuration [33]), which is shown in Fig. 5 (a). We point out that the convective cell width is comparable to the SOL width evaluated at the $\mathrm{X}$-point, i.e. retaining the effect of the flux expansion. In agreement with our simulation, experimental observations suggest that the $\mathbf{E} \times \mathbf{B}$ drift in $\mathrm{SF}$ configurations provides an important transport mechanism in the null-region and contributes to the heat flux distribution among the four divertor legs [34]. This mechanism has also been pointed out by measurements in the null-region of SN configurations $[35,36]$. We note that Refs. [37, 38] argue that an electromagnetic instability, the churning mode, may provide the redistribution of the heat flux over the four divertor legs. Our simulation shows that the redistribution occurs also when electromagnetic fluctuations are not included.

In the $\mathrm{SF}+$, the $\overline{\mathbf{v}}_{\mathrm{E}}$ drift in the region between the two X-points activates SP2 and SP3 [see Fig. 5 (b)]. However, the equilibrium parallel heat flux on SP2 and SP3 is reduced with respect to the ideal SF. This reminds of experimental observations in TCV [6] that show a decrease of the heat flux on the secondary strike points (SP2 and SP3) as the distance between the two X-points increases. We highlight that, in our simulation, the distance between the two X-points is comparable to the SOL width evaluated at the primary $\mathrm{X}$-point, corresponding to the width of the convective cell [see Fig. 5 (b)]. This is sufficient to lead to a reduction of $40 \%$ of the flux to SP2. In order to have significant activation of SP2 and SP3, we expect that the vertical distance between the two X-points 


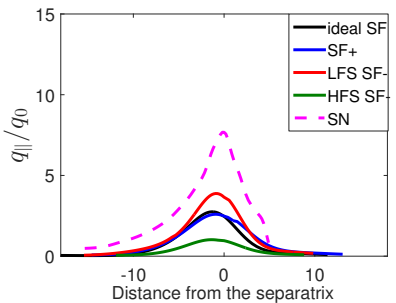

(a) $S P 1$

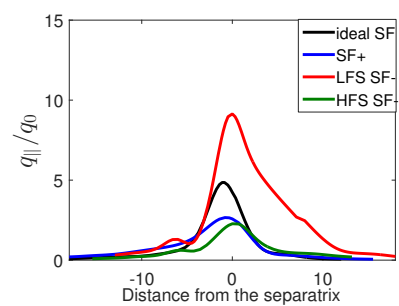

(b) SP2

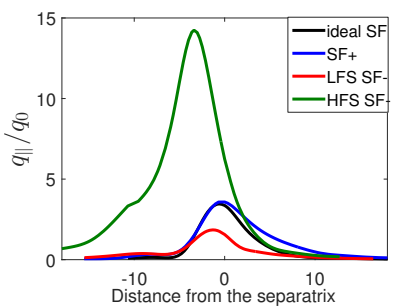

(c) $\mathrm{SP}_{3}$

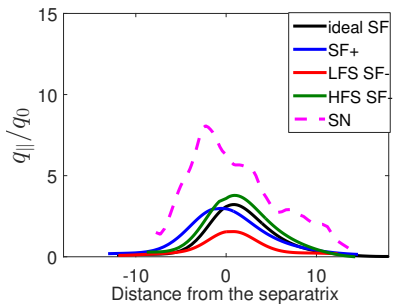

(d) $\mathrm{SP}_{4}$

Figure 4: Equilibrium parallel heat flux at the target plates, normalized to $q_{0}=n_{0} T_{e 0} c_{s 0}$, as a function of the distance from the separatrix, which is evaluated at the midplane and normalized to $\rho_{s 0}$. The position of the strike points is shown in Fig. 1 (solid white lines). The positive (negative) sign of the distance refers to a region to the right (left) of the separatrix.

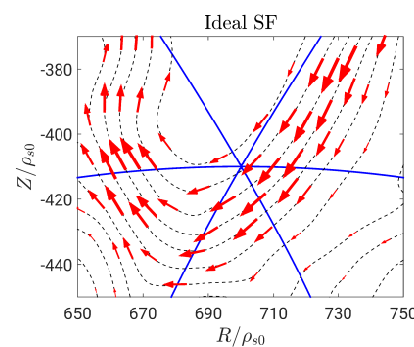

(a) Ideal $S F$

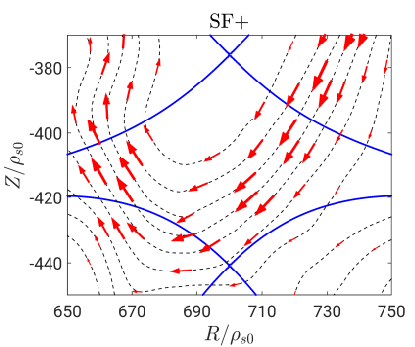

(b) $S F+$

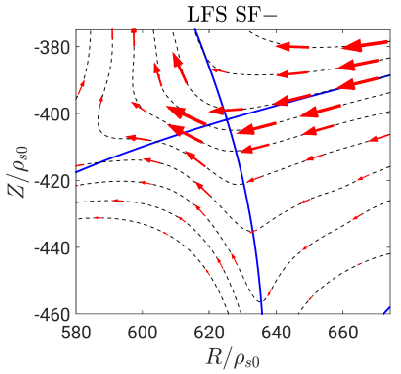

(a) Primary X-point

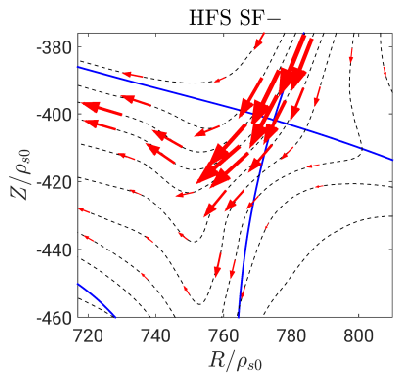

(c) Primary $X$-point

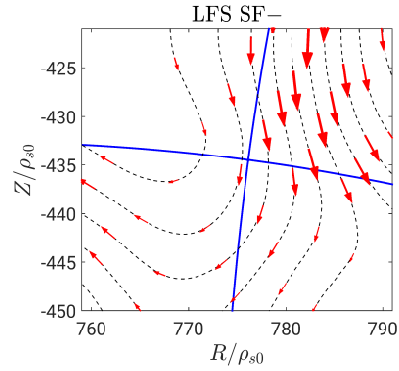

(b) Secondary X-point

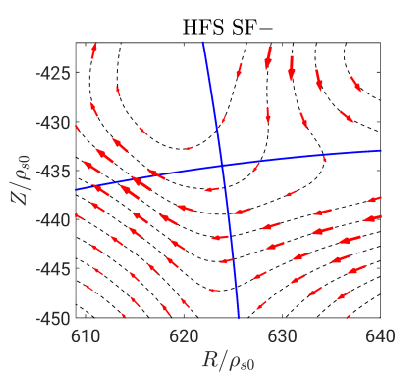

(d) Secondary X-point

primary X-point. Such a request may be challenging for DEMO, whose SOL width is foreseen to be smaller than $1 \mathrm{~mm}$ [39].

Regarding the LFS SF - configuration, we notice that the legs connected to the secondary X-point are only partially activated, i.e. only $9 \%$ and $10 \%$ of the total power is deposited on SP3 and SP4, respectively. In Fig. 6 , we show $\mathbf{q}_{\mathrm{E}}^{\text {eq }}$ around the primary (a) and secondary (b) X-point. The intensity of the circulation cell around the secondary X-point is one order of magnitude smaller than around the primary $\mathrm{X}$-point and $\mathbf{q}_{\mathrm{E}}^{\mathrm{eq}}$ is localized around the $\mathrm{X}$-point in a region of size smaller than the SOL width. It follows that, since the distance between the two Xpoints is approximately twice as large as the SOL width evaluated at the primary $\mathrm{X}$-point, the convective cell around the primary $\mathrm{X}$-point does not affect the heat fluxes in the proximity of the secondary X-point.

We now focus on the distribution of the heat flux between the LFS strike points (SP2, SP3, and SP4),

Figure 6: Equilibrium $\mathbf{E} \times \mathbf{B}$ heat flux for the LFS (top line) and HFS (bottom line) SF- configurations around the primary $[(a)$ and (c)] and secondary [(b) and (d)] X-points. The size of the arrows is proportional to the intensity of the heat flux. Black dashed lines represent the contour levels of the electrostatic potential. The separatrix is indicated by a solid blue line. In plot (b) and (d), the arrow size is multiplied by a factor of 10 with respect to (a) and (c).

more precisely between the LFS strike point connected to the primary X-point, i.e. SP2, and the strike points connected to the secondary X-point, i.e. SP3 and SP4. For this purpose, we first consider $q_{\|}$at the LFS midplane [7], a region where the parallel flow is directed towards the LFS targets. We integrate $q_{\|}$over the SOL region between the primary and the secondary 
separatrix and over the whole SOL region. The ratio between these two integrals, equal to 0.87 , is different from the ratio of the integrated power reaching SP2 to the power reaching the three LFS strike points, which is approximately 0.74. This is due to the turbulent $\mathbf{E} \times \mathbf{B}$ transport, $\mathbf{q}_{\mathrm{E}}^{\mathrm{t}}=\overline{\widetilde{p}_{e} \widetilde{\mathbf{v}}_{\mathrm{E}}}$, present in the region between the two separatrices on the LFS. In Fig. 7 (a), we show the intensity of the cross-field turbulent transport, $\mathbf{q}_{\mathrm{E}}^{\mathrm{t}} \cdot \nabla \psi /\|\nabla \psi\|$, in the LFS SF- configuration, with the dashed black line that encloses the region of strong perpendicular turbulent transport. As a result of the enhanced turbulence, heat is transported across the secondary separatrix to the secondary X-point. Due to the presence of the small but not completely negligible $\mathbf{E} \times \mathbf{B}$ convective cell around the secondary X-point, the heat flux then flows towards SP3 and SP4, while the power flowing to SP2 decreases. We note that the presence of turbulent structures in the region between the two nulls of the LFS SF - has been experimentally observed in Ref. [40]. On the other hand, $\mathbf{q}_{\mathrm{E}}^{\mathrm{eq}}$ is important only in the region around the primary Xpoint and cannot provide a mechanism to transport heat across the secondary separatrix. Moreover, there is no strong perpendicular equilibrium $\mathbf{E} \times \mathbf{B}$ flux that connects the two X-points. This explains the small heat flux exhausted at SP3 and SP4.

We now consider the HFS SF- configuration. We notice that SP1 and SP2 are only partially activated (see Fig. 4) and most of the power (60\%) flows to SP3. In this case, a very strong equilibrium $\mathbf{E} \times \mathbf{B}$ drift is found in the region around the primary X-point [see Fig. 6 (c)], such that it leads to a strong heat transport from the leg connected to SP4 to the leg connected to SP3. Around the secondary $\mathrm{X}$-point $\mathrm{q}_{\mathrm{E}}^{\mathrm{eq}}$ is more than one order of magnitude smaller [see Fig. 6 (d)] and therefore its role is negligible. Analogously to the LFS SF - configuration, the secondary X-point splits the SOL in two regions. By integrating $q_{\|}$at the HFS midplane, where the parallel heat fluxes are directed towards the HFS targets, over the region inside and outside the secondary separatrix, we expect that $85 \%$ of the total HFS power flows towards SP3. In fact, the integrated power on SP3 normalized to the total power flowing in the HFS SOL and reaching the HFS strike points (SP1, SP2, and SP3) is approximately $81 \%$, in good agreement with the expected value. As shown in Fig. 7, in the case of HFS SF- (b), there is no strong perpendicular turbulent transport between the two separatrices, in contrast to the observations in the LFS SF- (a). The fact that the perpendicular turbulent transport is enhanced only on the LFS of the LFS SF-, where large radial pressure gradients exist in the bad curvature region, indicates that it is driven by ballooning-like modes. This result confirms the hypothesis of Ref. [8], where the measured power on

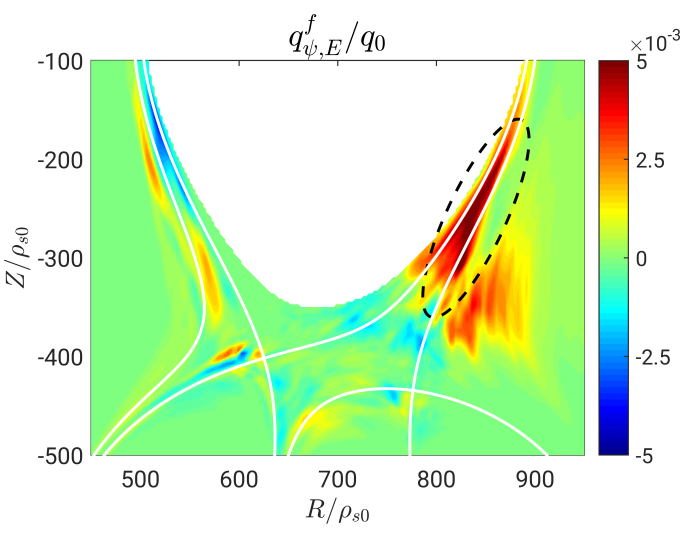

(a) LFS SF-

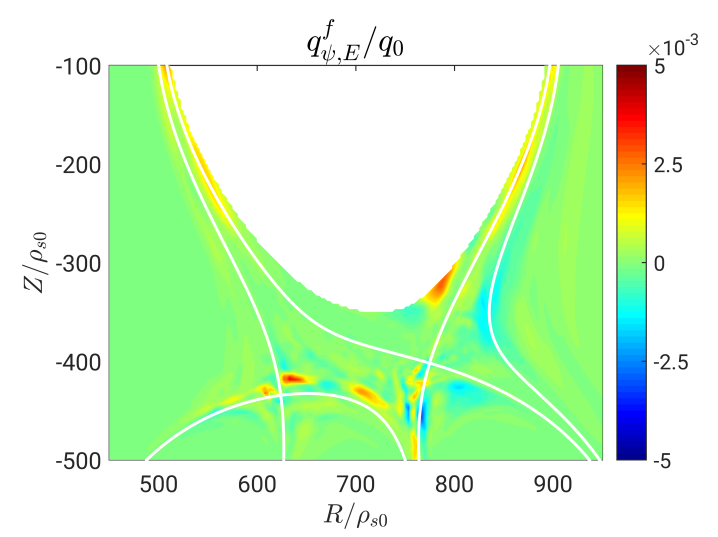

(b) HFS SF-

Figure 7: Intensity of the $\mathbf{E} \times \mathbf{B}$ turbulent flux projected along $\nabla \psi, q_{\psi, E}^{f}$, in the LFS and HFS SFconfigurations normalized to $q_{0}=n T_{e 0} c_{s 0}[(a)$ and (b) respectively]. The dashed black line highlights the region of strong turbulent transport. The white solid lines represents the two separatrices.

SP4 was found to be significantly larger than expected, and ballooning-like turbulent transport in the SOL between the primary and secondary separatrices was suggested as possible explanation.

For both the SF- configurations, the distance between the two separatrices can be optimized in order to achieve a better heat flux redistribution $[7,41]$. The optimization requires that the distance between the primary and secondary separatrix is set to fractions of the SOL width. The enhancement of perpendicular turbulent transport for the LFS SF- increases the distance at which the uniform heat flux distribution is reached, which is particularly beneficial for DEMOlike reactors. On the other hand, the width of the $\mathbf{E} \times \mathbf{B}$ convection cell around the primary $\mathrm{X}$ point is approximately a factor of 2 smaller in SFconfigurations than in the other SF configurations, decreasing the potential importance of $\mathrm{q}_{\mathrm{E}}^{\mathrm{eq}}$.

Finally, we compare the parallel heat flux for the 
SF and SN configurations. The peak value of the parallel heat flux in the SN configuration is more than twice as high as the one in the ideal SF (Fig. 4), as a consequence of the uniform parallel heat flux distribution among all the four divertor legs. This highlights the benefit of the heat flux redistribution. The same is valid for the $\mathrm{SF}+$, although the integrated power in SP2 and SP3 is reduced with respect to the ideal case. In the case of the LFS SF- configuration, the peak value of the parallel heat flux, which occurs in SP2, is comparable with the peak value of the parallel heat flux in the SN configuration. This is a consequence of the fact that in the LFS SFconfiguration the heat flux is not as well distributed among all the strike points. Worst considerations can be drawn for the HFS SF-, where the peak value of the parallel heat flux, which occurs in SP3, is $50 \%$ larger than the one in the SN.

As a conclusion of the present work, we remark that alternative exhaust configurations can now be studied by means of first-principles global turbulence simulations. The first simulations, carried out in SF configurations and described in the present Letter, point out that the activation of the secondary strike points (SP2 and SP3) in the ideal SF and the SF+ configurations, experimentally observed in Ref. [6], can be explained by the presence of an equilibrium $\mathbf{E} \times \mathbf{B}$ convective cell in the region around the primary Xpoint, a feature that may also be captured by multifluid codes that include equilibrium drifts [42]. The effect of the convective cell is strongly reduced as the distance between the two X-points becomes larger than the SOL width evaluated at the primary Xpoint, which is the case for our SF- configurations. Thanks to our turbulence simulations, it was possible to identify for the LFS SF - the presence of a region of enhanced cross-field turbulent transport driven by ballooning-like modes, thus confirming the hypothesis in Ref. [8] based on experimental observations. As a future work, we plan to investigate the origin of the convective $\mathbf{E} \times \mathbf{B}$ cell around the $\mathrm{X}$-point, the effect of the toroidal magnetic field direction on the parallel heat flux distribution among the strike points and the possible advantages of detachment in SF configurations. We will also carry out simulations removing the Boussinesq approximation in order to evaluate its effect on the parallel heat flux distribution among the four strike points. The presence and the role of the churning mode will be addressed by means of future simulations that include electromagnetic fluctuations.

\section{Acknowledgments}

This work was carried out in the framework of a multi team effort focused on the simulation of alternative divertor configurations within the EUROfusion work package WP-DTT1/ADC led by F. Militello and supported by the EUROfusion - Theory and Advanced Simulation Coordination (E-TASC). The authors thank J. Boedo, B. Labit, R. Maurizio, H. Reimerdes, C. Theiler, and C. Tsui for useful discussions. The simulations presented herein were carried out in part at the Swiss National Supercomputing Center (CSCS) under the project ID $\mathrm{s} 882$ and in part on the CINECA Marconi supercomputer under the GBSedge project. This work was supported in part by the Swiss National Science Foundation, was carried out within the framework of the EUROfusion Consortium and has received funding from the Euratom research and training programme 2014 - 2018 and 2019 - 2020 under grant agreement No 633053. The views and opinions expressed herein do not necessarily reflect those of the European Commission.

\section{References}

[1] H. Zohm, C. Angioni, E. Fable, G. Federici, G. Gantenbein, T. Hartmann, K. Lackner, E. Poli, L. Porte, O. Sauter, G. Tardini, D. Ward, and M. Wischmeier. On the physics guidelines for a tokamak DEMO. Nucl. Fusion, 53(7):073019, 2013.

[2] A. Loarte, B. Lipschultz, A.S Kukushkin, G.F Matthews, P.C Stangeby, N. Asakura, G.F Counsell, G. Federici, A. Kallenbach, K. Krieger, A. Mahdavi, V. Philipps, D. Reiter, J. Roth, J. Strachan, D. Whyte, R. Doerner, T. Eich, W. Fundamenski, A. Herrmann, M. Fenstermacher, P. Ghendrih, M. Groth, A. Kirschner, S. Konoshima, B LaBombard, P. Lang, A.W Leonard, P. Monier-Garbet, R. Neu, H. Pacher, B. Pegourie, R.A Pitts, S. Takamura, J. Terry, E. Tsitrone, the ITPA Scrape-off Layer Group, and Diver. Chapter 4: Power and particle control. Nucl. Fusion, 47(6):S203-S263, 2007.

[3] N. Asakura, K. Shinya, K. Tobita, K. Hoshino, K. Shimizu, H. Utoh, Y. Someya, M. Nakamura, N. Ohno, M. Kobayashi, and H. Tanaka. Investigation of advanced divertor magnetic configuration for demo tokamak reactor. Fusion Sci. Technol., 63(1T):70-75, 2013.

[4] D. D. Ryutov. Geometrical properties of a snowflake divertor. Phys. Plasmas, 14(6):064502, 2007.

[5] F. Piras, S. Coda, B. P. Duval, B. Labit, J. Marki, S. Yu Medvedev, J.-M. Moret, A. Pitzschke, and O. Sauter. Snowflake $\mathrm{H}$ Mode in a Tokamak Plasma. Phys. Rev. Lett., 105(15):155003, 2010.

[6] H. Reimerdes, G. P. Canal, B. P. Duval, B. Labit, T. Lunt, W. A. J. Vijvers, S. Coda, G. De Temmerman, T. W. Morgan, F. Nespoli, and B. Tal. Power distribution in the snowflake divertor in TCV. Plasma Phys. Control. Fusion, 55(12):124027, 2013.

[7] B. Labit, G.P. Canal, N. Christen, B.P. Duval, B. Lipschultz, T. Lunt, F. Nespoli, H. Reimerdes, U. Sheikh, C. Theiler, C.K. Tsui, K. Verhaegh, and W.A.J. Vijvers. Experimental studies of the snowflake divertor in TCV. Nucl. Mater. Energy, 12:1015-1019, 2017.

[8] R. Maurizio, C.K. Tsui, B.P. Duval, H. Reimerdes, 
C. Theiler, J. Boedo, B. Labit, U. Sheikh, and M. Spolaore. The effect of the secondary x-point on the scrape-off layer transport in the TCV snowflake minus divertor. Nucl. Fusion, 59(1):016014, 2019.

[9] V. A. Soukhanovskii, R. E. Bell, A. Diallo, S. Gerhardt, S. Kaye, E. Kolemen, B. P. LeBlanc, A. G. McLean, J. E. Menard, S. F. Paul, M. Podesta, R. Raman, T. D. Rognlien, A. L. Roquemore, D. D. Ryutov, F. Scotti, M. V. Umansky, D. Battaglia, M. G. Bell, D. A. Gates, R. Kaita, R. Maingi, D. Mueller, and S. A. Sabbagh. Snowflake divertor configuration studies in National Spherical Torus Experiment. Phys. Plasmas, 19(8):082504, 2012.

[10] V.A. Soukhanovskii, R.E. Bell, A. Diallo, S. Gerhardt, S. Kaye, E. Kolemen, B.P. LeBlanc, A. McLean, J.E. Menard, S.F. Paul, M. Podesta, R. Raman, D.D. Ryutov, F. Scotti, R. Kaita, R. Maingi, D.M. Mueller, A.L. Roquemore, H. Reimerdes, G.P. Canal, B. Labit, W. Vijvers, S. Coda, B.P. Duval, T. Morgan, J. Zielinski, G. De Temmerman, and B. Tal. Advanced divertor configurations with large flux expansion. J. Nucl. Mater., 438:S96-S101, 2013.

[11] V.A. Soukhanovskii, S.L. Allen, M.E. Fenstermacher, C.J. Lasnier, M.A. Makowski, A.G. McLean, W.H. Meyer, D.D. Ryutov, E. Kolemen, R.J. Groebner, A.W. Hyatt, A.W. Leonard, T.H. Osborne, T.W. Petrie, and J. Watkins. Developing physics basis for the snowflake divertor in the DIII-D tokamak. Nucl. Fusion, 58(3):036018, 2018.

[12] T. Lunt, G. P. Canal, Y. Feng, H. Reimerdes, B. P. Duval, B. Labit, W. A. J. Vijvers, D. Coster, K. Lackner, and M. Wischmeier. First EMC3-Eirene simulations of the TCV snowflake divertor. Plasma Phys. Control. Fusion, 56(3):035009, 2014.

[13] T. Lunt, G. P. Canal, B. P. Duval, Y. Feng, B. Labit, P. McCarthy, H. Reimerdes, W. A. J. Vijvers, and M. Wischmeier. Numerical study of potential heat flux mitigation effects in the tcv snowflake divertor. Plasma Phys. Control. Fusion, 58(4):045027, 2016.

[14] O. Pan, T. Lunt, M. Wischmeier, and D. Coster. SOLPS simulations of detachment in a snowflake configuration for the future upper divertor in ASDEX Upgrade. Plasma Phys. Control. Fusion, 60(8):085005, 2018.

[15] P. Ricci, F. D. Halpern, S. Jolliet, J. Loizu, A. Mosetto, A. Fasoli, I. Furno, and C. Theiler. Simulation of plasma turbulence in scrape-off layer conditions: the GBS code, simulation results and code validation. Plasma Phys. Control. Fusion, 54(12):124047, 2012.

[16] F.D. Halpern, P. Ricci, S. Jolliet, J. Loizu, J. Morales, A. Mosetto, F. Musil, F. Riva, T.M. Tran, and C. Wersal. The GBS code for tokamak scrape-off layer simulations. J. Comput. Phys., 315:388-408, 2016.

[17] P. Paruta, P. Ricci, F. Riva, C. Wersal, C. Beadle, and B. Frei. Simulation of plasma turbulence in the periphery of diverted tokamak by using the GBS code. Phys. Plasmas, 25(11):112301, 2018.

[18] B. D. Dudson, A. Allen, G. Breyiannis, E. Brugger, J. Buchanan, L. Easy, S. Farley, I. Joseph, M. Kim, A. D. McGann, J. T. Omotani, M. V. Umansky, N. R. Walkden, T. Xia, and X. Q. Xu. BOUT++: Recent and current developments. J. Plasma Phys., 81(1):365810104, 2015.

[19] B. Zhu, M. Francisquez, and B. N. Rogers. GDB: A global 3D two-fluid model of plasma turbulence and transport in the tokamak edge. Comput. Phys. Commun., 232:46$58,2018$.

[20] A. Stegmeir, D. Coster, A. Ross, O. Maj, K. Lackner, and E. Poli. GRILLIX: a 3D turbulence code based on the flux-coordinate independent approach. Plasma Phys. Control. Fusion, 60(3):035005, 2018.
[21] A.H. Nielsen, G.S. Xu, J. Madsen, V. Naulin, J. Juul Rasmussen, and B.N. Wan. Simulation of transition dynamics to high confinement in fusion plasmas. Phys. Lett. A, 379(47-48):3097-3101, 2015.

[22] P. Tamain, H. Bufferand, G. Ciraolo, C. Colin, D. Galassi, $\mathrm{Ph}$ Ghendrih, F. Schwander, and E. Serre. The TOKAM3X code for edge turbulence fluid simulations of tokamak plasmas in versatile magnetic geometries. $J$. Comput. Phys., 321:606-623, 2016.

[23] A. Zeiler, J. F. Drake, and B. Rogers. Nonlinear reduced Braginskii equations with ion thermal dynamics in toroidal plasma. Phys. Plasmas, 4(6):2134-2138, 1997.

[24] G. Q. Yu, S. I. Krasheninnikov, and P. N. Guzdar. Twodimensional modelling of blob dynamics in tokamak edge plasmas. Phys. Plasmas, 13(4):042508, 2006.

[25] A. Stegmeir, A. Ross, T. Body, M. Francisquez, W. Zholobenko, D. Coster, O. Maj, P. Manz, F. Jenko, B. N. Rogers, and K. S. Kang. Global turbulence simulations of the tokamak edge region with GRILLIX. Phys. Plasmas, 26(5):052517, 2019.

[26] A. Ross, A. Stegmeir, P. Manz, D. Groselj, W. Zholobenko, D. Coster, and F. Jenko. On the nature of blob propagation and generation in the large plasma device: Global GRILLIX studies. Phys. Plasmas, 26(10):102308, 2019.

[27] K. Bodi, G. Ciraolo, Ph. Ghendrih, F. Schwander, E. Serre, and P. Tamain. Impact of the Boussinesq approximation in tokamak scrape-off layer turbulence. In 38th EPS Conference on Plasma Physics P1.121, Strasbourg, France, 2011.

[28] S. Jolliet, F. D. Halpern, J. Loizu, A. Mosetto, and P. Ricci. Aspect ratio effects on limited scrape-off layer plasma turbulence. Phys. Plasmas, 21(2):022303, 2014.

[29] T. Body, A. Stegmeir, W. Zholobenko, D. Coster, and F. Jenko. Treatment of advanced divertor configurations in the fluxcoordinate independent turbulence code GRILLIX. Contrib. Plasma Phys., e201900139, 2019.

[30] M. Fontana, L. Porte, S. Coda, O. Sauter, TCV Team, et al. The effect of triangularity on fluctuations in a tokamak plasma. Nucl. Fusion, 58(2):024002, 2017.

[31] J. Loizu, P. Ricci, F. D. Halpern, and S. Jolliet. Boundary conditions for plasma fluid models at the magnetic presheath entrance. Phys. Plasmas, 19(12):122307, 2012.

[32] P. C. Stangeby. The plasma boundary of magnetic fusion devices. CRC Press, 2000.

[33] N.R. Walkden, J. Harrison, S.A. Silburn, T. Farley, S.S. Henderson, A. Kirk, F. Militello, and A. Thornton. Quiescence near the X-point of MAST measured by high speed visible imaging. Nucl. Fusion, 57(12):126028, 2017.

[34] G.P. Canal, T. Lunt, H. Reimerdes, B.P. Duval, B. Labit, and W.A.J. Vijvers. Enhanced $E \times B$ drift effects in the TCV snowflake divertor. Nucl. Fusion, 55(12):123023, 2015.

[35] M.J. Schaffer, J.A. Boedo, R.A. Moyer, T.N. Carlstrom, and J.G. Watkins. Large $\mathrm{E} \times \mathrm{B}$ convection near the divertor X-point. J. Nucl. Mater., 290-293:530-536, 2001.

[36] M. J. Schaffer, B. D. Bray, J. A. Boedo, T. N. Carlstrom, R. J. Colchin, C. L. Hsieh, R. A. Moyer, G. D. Porter, T. D. Rognlien, and J. G. Watkins. ExB circulation at the tokamak divertor $\mathrm{X}$ point. Phys. Plasmas, 8(5):2118-2124, 2001.

[37] D. D. Ryutov, R. H. Cohen, W. A. Farmer, T. D. Rognlien, and M. V. Umansky. The churning mode' of plasma convection in the tokamak divertor region. Phys. Scr., 89(8):088002, 2014.

[38] M. V. Umansky and D. D. Ryutov. Toroidally symmetric plasma vortex at tokamak divertor null point. Phys. 
Plasmas, 23(3):030701, 2016.

[39] T. Eich, A. W. Leonard, R. A. Pitts, W. Fundamenski, R. J. Goldston, T. K. Gray, A. Herrmann, A. Kirk, A. Kallenbach, O. Kardaun, et al. Scaling of the tokamak near the scrape-off layer $\mathrm{H}$-mode power width and implications for ITER. Nucl. Fusion, 53(9):093031, 2013.

[40] N. R. Walkden, B. Labit, H. Reimerdes, J. Harrison, T. Farley, P. Innocente, and F. Militello. Fluctuation characteristics of the TCV snowflake divertor measured with high speed visible imaging. Plasma Phys. Control. Fusion, 60(11):115008, 2018

[41] P. J. Vail, O. Izacard, and E. Kolemen. Optimization of the snowflake divertor for power and particle exhaust on NSTXU. Nucl. Mater. Energy, 19:516-523, 2019.

[42] R. Schneider, X. Bonnin, K. Borrass, D.P. Coster, H. Kastelewicz, D. Reiter, V.A. Rozhansky, and B.J. Braams. Plasma edge physics with B2-eirene. Contributions to Plasma Physics, 46(1-2):3-191, 2006. 